\title{
Hemi-laryngopharyngeal spasm as a novel cause of inducible laryngeal obstruction with a surgical cure: report of 3 cases
}

\author{
Christopher R. Honey, MD, DPhil, ${ }^{1}$ Murray D. Morrison, MD, ${ }^{2}$ Manraj K. S. Heran, MD, ${ }^{3}$ and \\ Baljinder S. Dhaliwal, MD4
}

Divisions of ${ }^{1}$ Neurosurgery and ${ }^{2}$ Otolaryngology, Department of Surgery; and Departments of ${ }^{3}$ Radiology and ${ }^{4}$ Anesthesiology, Pharmacology and Therapeutics at the University of British Columbia, Vancouver, British Columbia, Canada

Inducible laryngeal obstruction has been described under at least 40 different monikers, including vocal cord dysfunction, paroxysmal vocal fold motion, and irritable larynx. The etiology of this condition is believed to be laryngeal hyperactivity in response to psychological issues or acid reflux. Most patients are treated with some combination of proton pump inhibitors, speech therapy, and psychotherapy. However, a small cohort of patients remains refractory to all medical interventions. The authors describe a novel condition, hemi-laryngopharyngeal spasm (HELPS), which can cause severe episodic stridor leading to unconsciousness in association with cough. The first recognized and surgically cured patient with HELPS was reported in an earlier issue of this journal. Three additional patients have been followed up for at least a year postoperatively, and their cases are reported here.

Each patient presented with a similar pattern of episodic coughing and choking that increased in frequency, severity, and duration over years. The episodes eventually occurred while sleeping and could cause severe stridor with loss of consciousness. All three patients were initially misdiagnosed with a psychiatric illness and subjected to multiple intubations and one tracheostomy. Unilateral botulinum toxin injections in the vocal fold eased the severity of the throat contractions but not the cough. Magnetic resonance imaging showed a looping posterior inferior cerebellar artery juxtaposed to a vagus nerve in each case. Microvascular decompression (MVD) of that vessel relieved all symptoms.

The introduction of this new medical condition may help a small cohort of patients with inducible laryngeal obstructions that have not responded to the current standard treatments. Patients are asymptomatic between episodes of progressively severe coughing and choking with stridor that may lead to intubation. Severe anxiety about the unpredictable symptoms is expected and may contribute to a psychiatric misdiagnosis. Microvascular decompression for HELPS is more difficult than that for trigeminal neuralgia because the involved nerve is more susceptible to manipulation. Ultimately, the final proof that HELPS is a real and distinct syndrome will require its recognition and successful treatment by colleagues around the world.

https://thejns.org/doi/abs/10.3171/2018.2.JNS172952

KEYWORDS hemi-laryngopharyngeal spasm; HELPS; vocal cord dysfunction; paradoxical vocal fold motion; inducible laryngeal obstruction; microvascular decompression; pain

$\mathrm{T}$ HE discovery of a medical condition offers new hope to those who have been previously misdiagnosed and can now be cured. Conversely, the new treatment may cause unnecessary complications for those erroneously diagnosed during the early years of understanding a condition.
Hemi-laryngopharyngeal spasm (HELPS) is a recognizable, curable, but relatively uncommon condition. In the last 3 years, we have operated on five patients with the disorder: the first with a 3 -year follow-up, ${ }^{6}$ three with more than 1 year of follow-up each (subjects of this paper), and one with a shorter follow-up. These numbers likely re-

ABBREVIATIONS CN = cranial nerve; GPN = glossopharyngeal neuralgia; HELPS = hemi-laryngopharyngeal spasm; HFS = hemifacial spasm; MVD = microvascular decompression; PICA = posterior inferior cerebellar artery; PVFM = paradoxical vocal fold motion; VCD = vocal cord dysfunction. ACCOMPANYING EDITORIAL See pp 1862-1864. DOI: 10.3171/2018.3.JNS18385.

SUBMITTED November 27, 2017. ACCEPTED February 8, 2018.

INCLUDE WHEN CITING Published online July 20, 2018; DOI: 10.3171/2018.2.JNS172952. 
flect the prevalence of the condition, and its true incidence remains unknown. During the same period, we have operated on only three patients with glossopharyngeal neuralgia (GPN). All patients with both of these conditions within the province of British Columbia would probably be treated by the senior author given the nature of our referral pattern. We tentatively estimate that the incidence of HELPS is slightly more than that of GPN,${ }^{8}$ or greater than 1 case per 100,000 people per year.

What has happened to all these patients before now? Case reports of patients with similar symptomatology, including those requiring tracheostomy ${ }_{1}^{14}$ have appeared under a variety of labels describing episodic laryngospasm. The most common terms are "vocal cord dysfunction" $(\mathrm{VCD})^{1}$ and "paradoxical vocal fold motion" (PVFM), ${ }^{11}$ but there are at least 40 different monikers describing similar but much less severe symptomatology. ${ }^{2}$ Normally, the larynx will reflexively contract to protect the lower airway from noxious irritants. In patients with VCD or PVFM, this normal function becomes hyperactive in response to psychological issues or acid reflux. The vast majority of these patients respond to appropriate speech therapy, ${ }^{15}$ psychotherapy, ${ }^{5}$ and proton pump blockers. ${ }^{9}$ A very small minority of nonresponders, however, may represent an untreated cohort with HELPS.

We now recognize that each patient had a similar core of symptoms including progressive, episodic throat contractions (choking) and coughing. Untreated, these symptoms progressed to occur while sleeping and led to a rare loss of consciousness. No patient reported unilateral throat pain (GPN). Whether the additional symptoms of episodic vocal changes, episodic sensation of an enlarged tongue, ${ }^{6}$ and globus reflect individual variations or are unrelated will become clear with additional cases. This case report highlights three patients with HELPS, describing their common features, diagnostic findings, and surgical treatment and highlighting the prevalence of this condition. This work was approved by the University of British Columbia Clinical Research Ethics Board, and written consent was provided by each patient for publication of their case.

\section{Case Reports \\ Case 1}

\section{History and Examination}

A 65-year-old man presented with a 5-year history of episodic but progressive throat contractions and coughing. The initial onset was insidious and not related to an upper respiratory tract infection or trauma. There was no recognized trigger for the throat contractions; they occurred spontaneously with increasing frequency, duration, and intensity over the years. Some contractions could be felt asymmetrically on the left side, but others were symmetrical. The contractions began to occur during sleep and would typically last 15 minutes, although several could occur in a row with a few minutes between bouts. The contractions caused severe stridor and an intense choking sensation that was frightening and anxiety provoking. The contractions would stop spontaneously, usually before he reached the emergency room by ambulance, and his sub- sequent examination was reported to be entirely normal. The etiology was believed to be psychogenic until several witnessed episodes resulted in loss of consciousness. Later, the choking episodes were accompanied by progressively severe coughing. The coughing was triggered by a tickling sensation deep to his suprasternal notch and would last longer than the choking. Eventually, the coughing also occurred independently and while he was sleeping. The coughing was intense enough to cause visual phosphenes and severe tussive headaches. Investigations revealed asthma and acid reflex, and he had been aggressively treated for both with no improvement. Symptoms continued despite therapeutic dosages of omeprazole, amitriptyline, domperidone, montelukast, cetirizine, fluticasone, salmeterol, and tiotropium. A tracheostomy was placed so he could breathe during the choking episodes. Left-sided botulinum toxin (Botox) injections in his larynx reduced the severity of the contractions by approximately $80 \%$ but did not change the frequency or duration of choking and had no effect on the coughing. Magnetic resonance imaging (Fig. 1A and $\mathrm{B}$ ) demonstrated the posterior inferior cerebellar artery (PICA) curving into the vicinity of the left cranial nerve $(\mathrm{CN}) \mathrm{X}$ root entry zone, but the nerve rootlets were not well visualized.

\section{Operation}

Neurosurgical microvascular decompression (MVD) of the left vagus nerve was performed with the patient under general anesthesia delivered through his tracheostomy with simultaneous visualization of his larynx with a bronchoscope. Looking between the six rootlets of the vagus nerve, the PICA was found curving into the caudal rootlets (Video 1 for intraoperative video).

VIDEO 1. Intraoperative microscopy during MVD for each patient. Case 1. The left PICA is looping against and compressing the caudal rootlets of the vagus nerve and is eventually mobilized anterocaudally. Case 2. The right PICA is compressing the more rostral rootlets of the vagus nerve and is mobilized antero-caudally. Case 3. The left PICA is compressing the caudal rootlets of the vagus nerve and is mobilized antero-caudally. Copyright Christopher R. Honey. Published with permission. Click here to view.

The vessel was surgically displaced antero-caudally with a pledget of shredded surgical felt (Bard PV, USA) and Tisseel (Baxter, Canada) to decompress the nerve. Intraoperative stimulation of the upper three vagus nerve rootlets caused no contractions of the throat. Stimulation of the lower three rootlets caused different contraction patterns of the vocal cords and throat (Video 2 for video laryngoscopy).

VIDEO 2. Case 1. Intraoperative video laryngoscopy. The patient was ventilated through his tracheostomy during surgery, which allowed for simultaneous visualization of his laryngopharynx during direct stimulation $(0.02 \mathrm{~mA}, 1 \mathrm{msec}, 2 \mathrm{~Hz})$ of each left vagus nerve rootlet. Stimulation of the rostral three rootlets (labeled rootlets 1, 2, and 3) caused no motor response. Stimulation of rootlet 4 caused ipsilateral vocal cord contraction. Stimulation of rootlet 5 caused ipsilateral vocal cord and pharynx contraction. Stimulation of rootlet 6 caused strong ipsilateral pharynx contraction. As documented in Video 1, the left PICA vessel primarily impacts the most caudal vagus nerve rootlet (rootlet 6 ), which may be why the patient was able to localize his throat contractions to the left side. Copyright Christopher R. Honey. Published with permission. Click here to view. 

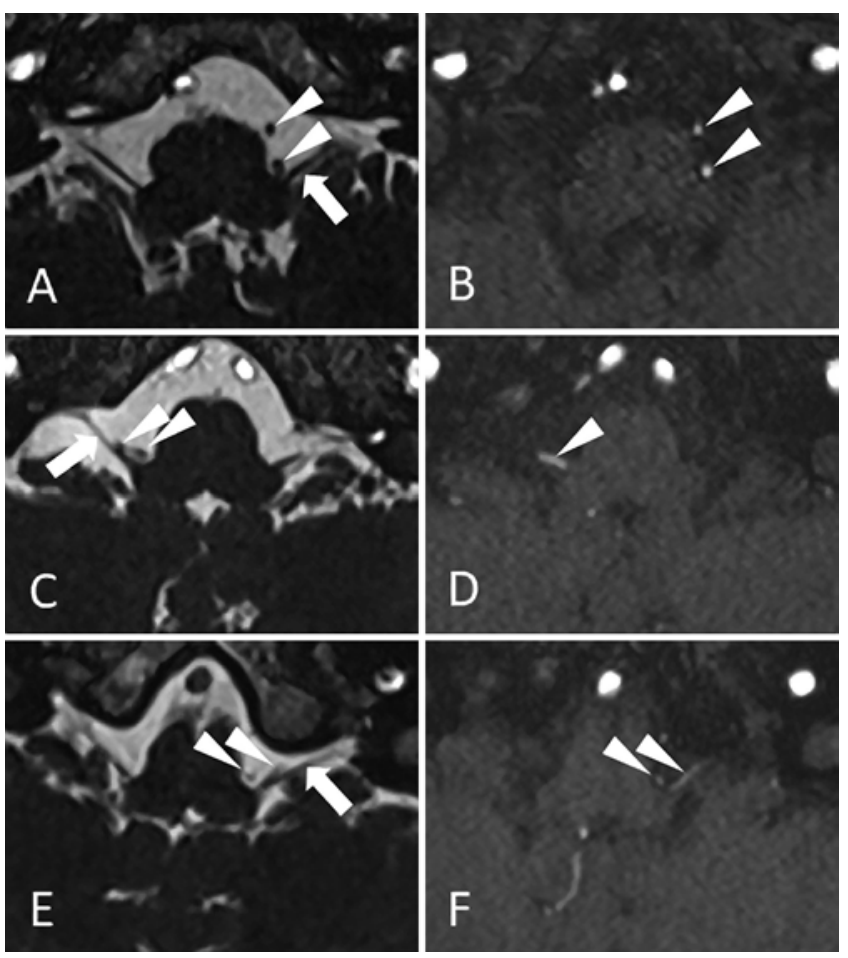

FIG. 1. Preoperative 1.5-T MRI. Axial constructive interference in steady state (CISS) and time-of-flight (TOF) images obtained at the same level for each of the patients: case 1 ( $A$ and B), case 2 (C and D), and case 3 (E and F). Arrows point to CN X, and arrowheads point to the vessel. The vessel in each case was the PICA, which is looping with its convex apex juxtaposed to the nerve but does not obviously distort the nerve on these sequences.

\section{Postoperative Course}

Postoperatively, the patient had immediate and complete relief of his symptoms. The immediate benefits were thought to be due to surgical injury of the nerve because after the surgery he had a breathy voice with reduced left vocal cord movement for 2 weeks and dysphagia for 2 months. These temporary complications completely resolved, and his choking and severe coughing had not recurred at 18 months' follow-up. His recovery was interrupted by a delayed CSF leak that required reclosure of the dura with a fascial graft. His tracheostomy was removed after 3 months.

\section{Case 2}

History and Examination

A 43-year-old woman presented with a 5-year history of episodic but progressive choking, coughing, and vocal changes. The symptoms began spontaneously. Initially she felt a mild midline throat tightening that would trigger a brief cough. The symptoms slowly progressed and began to occur while sleeping. She had a history of asthma since childhood, which had always been effectively treated with bronchodilators and could clearly be distinguished by the patient from her current HELPS symptoms. Trials of proton pump inhibitors and bronchodilators were unsuccessful, and chronic $\mathrm{pH}$ monitoring ruled out acid reflux.
Psychiatric consultation indicated that her anxiety and depression were more likely the result of her undiagnosed condition than the cause of it. The progressive throat contractions became circumferential and caused severe stridor leading to repeated loss of consciousness. The resultant coughing, which always accompanied the choking, was severe enough to cause stress incontinence and visual phosphenes. Following some episodes, she briefly felt an abnormal sensation on the right side of her throat, which she likened to a vibrating guitar string. Milder episodes of throat contractions, also felt as a circumferential tightness, could be triggered by prolonged or loud talking. When this occurred, the quality and strength of her voice would deteriorate to hoarseness initially then to hypophonia for 5-10 minutes. If the episode passed, her voice would return to normal; if the episode strengthened, she would have a stridor attack. Symptoms were refractory to pantoprazole, salbutamol, lorazepam, clonazepam, fluoxetine, and indacaterol-glycopyrronium. Magnetic resonance imaging (Fig. 1C and D) revealed a loop of PICA abutting the right CN X. Botox into the right vocal fold reduced the intensity of the stridor but not the cough.

\section{Operation}

Microvascular decompression was performed, and the rostral rootlets of the right vagus nerve were distorted posteriorly by a loop of PICA (Video 1). This anatomy was very similar to that in our first published case. ${ }^{1}$ The vessel was surgically displaced antero-caudally with felt and Tisseel.

\section{Postoperative Course}

Immediately following surgery, all her symptoms resolved and she was discharged home on the 2 nd day. She reported a weaker, breathy voice immediately after surgery, which returned to normal after 2 days. Four weeks after surgery, a milder version (less than half as intense) of her choking returned with no coughing. These residual symptoms gradually faded until she was asymptomatic at 4 months and remained so at her 1-year follow-up.

\section{Case 3}

\section{History and Examination}

A 48-year-old woman presented with a 10-year history of coughing and a 2-year history of choking. Her coughing began spontaneously and was initially ascribed to environmental allergens. Episodes were triggered by a sensation of tightness in the midline of her throat deep to the suprasternal notch. Over the years, the coughing became more frequent and intense, causing stress incontinence and vomiting. Episodes could be triggered by prolonged or loud use of her voice or any activity that increased her heart rate (especially going up stairs). Between episodes she had a mild globus sensation. Antihistamines, inhaled bronchodilators, oral steroids, and proton pump inhibitors were ineffective. Bronchoscopy and esophageal $\mathrm{pH}$ were normal. Episodes of throat contractions began more recently and progressed in frequency, duration, and severity. These episodes could follow a coughing fit or occur spontaneously. The resultant tightness in her throat was 
circumferential and not lateralized. Episodes began to occur while sleeping and caused frightening stridor with one episode of loss of consciousness. Botox injection into the left vocal fold dramatically reduced the choking intensity on two occasions but had no effect on the cough. Botox injection into the right vocal fold had no benefit. Magnetic resonance imaging (Fig. 1E and F) showed a looping PICA near the left CN X.

\section{Operation}

Microvascular decompression was performed, and the caudal rootlets of the left vagus nerve were directly impacted by a loop of the PICA (Video 1). This vessel was surgically displaced antero-caudally with felt and Tisseel.

\section{Postoperative Course}

Her globus sensation was immediately relieved, but her voice was weaker and breathy immediately following surgery. She did not have dysphagia and was discharged home on day 3. Her voice returned to normal after 1 week, and her choking and coughing stopped within 2 weeks and had not returned at the 1-year follow-up.

\section{Discussion}

Vascular compression of $\mathrm{CN}$ VII causes hemifacial spasm (HFS). ${ }^{7}$ We believe vascular compression of CN X causes HELPS. ${ }^{6}$ Both conditions cause progressive episodic symptoms, can occur during sleep, can be symptomatically treated with Botox, and can be cured by MVD of the compressed nerve. The pathophysiology underpinning both conditions is likely similar and may involve pressureinduced demyelination with ectopic generation and ephaptic transmission of impulses along the compressed nerve. ${ }^{13}$

The diagnosis of HELPS may be clear when a patient presents with episodic, unilateral throat contractions and an obvious vessel distorting the ipsilateral CN X. However, patients can report circumferential (nonlateralized) throat contractions (case 3), and vessel loops among the lower CNs are not uncommon. ${ }^{3} \mathrm{~A}$ definitive test is needed. Magnetic resonance imaging investigation of HELPS is hindered by poor visualization of CN X rootlets at a field strength of $1.5 \mathrm{~T}$, even with thin-cut heavily $\mathrm{T} 2$-weighted volumetric sequences (i.e., constructive interference in steady state [CISS] or fast imaging employing steady-state acquisition [FIESTA]). The rootlets are individually much smaller than CN V or VII. Video laryngoscopy during a spasm is difficult because such contractions are intermittent and patients are in extremis during an attack. The response to Botox injection is a promising test (and the subject of a prospective evaluation). Injecting the affected side produces a dramatic reduction in spasm intensity but no change in cough. Injecting the unaffected side in the patient in case 3 produced no benefit. This unilateral benefit may help to distinguish HELPS from other inducible laryngeal obstructions ${ }^{11}$ and guide the neurosurgical approach when symptoms are not obviously lateralized. We postulate that contraction of the pharynx may be felt as lateralized but contraction of the larynx may not.

The differential diagnosis of conditions causing episodic throat contractions includes allergic phenomena, laryngeal dystonia, ${ }^{4}$ gastroesophageal reflux disorder, vocal cord dysfunction, irritable larynx,${ }^{12}$ and now HELPS. There is no swelling of the tongue or throat with HELPS, and as soon as an episode is over, the patient's examination is normal. This distinguishes the disorder from allergy-mediated stridor. HELPS includes episodic coughing, which distinguishes it from laryngeal dystonia. HELPS does not respond to the standard treatments recommended for VCD or irritable larynx (e.g., proton pump inhibitors, speech therapy, and psychotherapy), and the symptoms typically progress to much more severe levels-all of our patients reported at least one attack that had caused brief unconsciousness. We do not know if this brief unconsciousness was due to hypoxia or cardiac arrhythmia (as is rarely reported during GPN). Unilateral Botox injections will ease the symptoms of VCD and irritable larynx, but the response should occur regardless of side-unlike the unilateral benefit in HELPS.

The neurosurgical treatment of HELPS requires experience with MVD. Our team has completed over 450 such operations without stroke or death. The surgical approach to $\mathrm{CN} X$ follows that for HFS around the inferolateral cerebellum. Surgeons should be aware that CN X does not tolerate as vigorous manipulation as $\mathrm{CN} \mathrm{V}$, and although sharp dissection between its rootlets to look for the offending vessel can be performed, ${ }^{6}$ retraction of the rootlets led to varying degrees of temporary dysphonia in all three of our patients and prolonged but temporary dysphagia in one. As in trigeminal neuralgia, compression of $\mathrm{CN}$ $\mathrm{X}$ can occur anywhere along its course (case 2), but less distortion may be required closer to the root entry zone (cases 1 and 3) where movement of the nerve is tethered. Pathological compression of the brainstem has also been reported to cause symptoms compatible with HELPS. ${ }^{10,16}$ The immediate but temporary benefit seen postoperatively in case 2 was followed by a slower but complete resolution of symptoms. This same phenomenon can occur following MVD for HFS or trigeminal neuralgia and likely represents a temporary microrhizotomy, followed by nerve remyelination.

There is a danger in over-diagnosing this new condition particularly when choking, coughing, and incidental vessels adjacent to the vagus nerve are common. Surgery to expose and decompress the vagus nerve rootlets is technically demanding and comes with significant risks. At this time, we would advocate surgery only for patients with 1) medically refractory symptoms interfering with their quality of life, 2) throat contractions that can be lateralized, 3) MRI studies demonstrating a vessel looping against the vagus nerve on the symptomatic side, and 4) a profound reduction $(>50 \%)$ in choking following Botox injection into the vocal fold only on the symptomatic side.

\section{Conclusions}

We hope that recognition of this new medical condition by our otolaryngology, emergency medicine, anesthesiology, respirology, psychiatry, neurology, family medicine, and neurosurgical colleagues will translate into better care for patients with HELPS. Patients presenting with episodic coughing and unilateral throat contractions should 
prompt MRI investigation for ipsilateral compression of the vagus nerve. Nonlateralized throat contractions may require more sophisticated investigations with sequential, alternating unilateral Botox injections in the vocal folds. During MVD of the vagus nerve, neurosurgeons should be aware that the nerve is far more sensitive to manipulation than the trigeminal nerve and that relatively minor manipulation led to temporary dysphonia in all of our patients. The final proof that HELPS is a real and distinct syndrome will require its recognition and successful treatment by colleagues around the world.

\section{Acknowledgments}

We thank the patients for their tenacity in searching for an answer to their illness, their bravery for agreeing to undergo this new surgery, and their honesty in reporting the results.

\section{References}

1. Altman KW, Mirza N, Ruiz C, Sataloff RT: Paradoxical vocal fold motion: presentation and treatment options. J Voice 14:99-103, 2000

2. Christensen PM, Heimdal JH, Christopher KL, Bucca C, Cantarella G, Friedrich G, et al: ERS/ELS/ACCP 2013 international consensus conference nomenclature on inducible laryngeal obstructions. Eur Respir Rev 24:445-450, 2015

3. Donahue JH, Ornan DA, Mukherjee S: Imaging of vascular compression syndromes. Radiol Clin North Am 55:123138,2017

4. Grillone GA, Blitzer A, Brin MF, Annino DJ Jr, Saint-Hilaire $\mathrm{MH}$ : Treatment of adductor laryngeal breathing dystonia with botulinum toxin type A. Laryngoscope 104:30-32, 1994

5. Guglani L, Atkinson S, Hosanagar A, Guglani L: A systematic review of psychological interventions for adult and pediatric patients with vocal cord dysfunction. Front Pediatr 2:82, 2014

6. Honey CR, Gooderham P, Morrison M, Ivanishvili Z: Episodic hemilaryngopharyngeal spasm (HELPS) syndrome: case report of a surgically treatable novel neuropathy. J Neurosurg 126:1653-1656, 2017

7. Jannetta PJ, Abbasy M, Maroon JC, Ramos FM, Albin MS: Etiology and definitive microsurgical treatment of hemifacial spasm. Operative techniques and results in 47 patients. $\mathbf{J}$ Neurosurg 47:321-328, 1977

8. Katusic S, Williams DB, Beard CM, Bergstralh EJ, Kurland LT: Epidemiology and clinical features of idiopathic trigeminal neuralgia and glossopharyngeal neuralgia: similarities and differences, Rochester, Minnesota, 1945-1984. Neuroepidemiology 10:276-281, 1991

9. Loughlin CJ, Koufman JA: Paroxysmal laryngospasm secondary to gastroesophageal reflux. Laryngoscope 106:15021505,1996
10. Maschka DA, Bauman NM, McCray PB Jr, Hoffman HT, Karnell MP, Smith RJ: A classification scheme for paradoxical vocal cord motion. Laryngoscope 107:1429-1435, 1997

11. Morris MJ, Christopher KL: Diagnostic criteria for the classification of vocal cord dysfunction. Chest 138:1213-1223, 2010

12. Morrison M, Rammage L, Emami AJ: The irritable larynx syndrome. J Voice 13:447-455, 1999

13. Nielsen VK: Electrophysiology of the facial nerve in hemifacial spasm: ectopic/ephaptic excitation. Muscle Nerve 8:545-555, 1985

14. Park DP, Ayres JG, McLeod DT, Mansur AH: Vocal cord dysfunction treated with long-term tracheostomy: 2 case studies. Ann Allergy Asthma Immunol 98:591-594, 2007

15. Patel RR, Venediktov R, Schooling T, Wang B: Evidencebased systematic review: effects of speech-language pathology treatment for individuals with paradoxical vocal fold motion. Am J Speech Lang Pathol 24:566-584, 2015

16. Patton H, DiBenedetto R, Downing E, Zoller M, Morgan K: Paradoxic vocal cord syndrome with surgical cure. South Med J 80:256-258, 1987

\section{Disclosures}

The authors report no conflict of interest concerning the materials or methods used in this study or the findings specified in this paper.

\section{Author Contributions}

Conception and design: Honey, Morrison. Acquisition of data: Honey, Morrison, Dhaliwal. Analysis and interpretation of data: Honey, Heran. Drafting the article: Honey. Critically revising the article: all authors. Reviewed submitted version of manuscript: all authors. Approved the final version of the manuscript on behalf of all authors: Honey.

\section{Supplemental Information \\ Videos}

Video 1. https://vimeo.com/266485308.

Video 2. https://vimeo.com/266485330.

\section{Previous Presentations}

Portions of this work were presented as an oral presentation at the World Society for Stereotactic and Functional Neurosurgery, Berlin, Germany, June 26-29, 2017.

\section{Correspondence}

Christopher R. Honey: University of British Columbia, Vancouver, BC, Canada. chris.honey@telus.net. 